\title{
Opportunities and Challenges in APT Metrology for Semiconductor Applications
}

Claudia Fleischmann ${ }^{1 *}$, Ramya Cuduvally ${ }^{1,2}$, Richard Morris ${ }^{1}$, Davit Melkonyan ${ }^{1,2}$, Jonathan Op de Beeck $^{1,2}$, Igor Makhotkin ${ }^{1,2}$, Paul van der Heide ${ }^{1}$ and Wilfried Vandervorst ${ }^{1,2}$

1. imec, Leuven, Belgium.

2. IKS, KU Leuven, Leuven, Belgium.

* Corresponding author: Claudia.Fleischmann@imec.be

For all emerging semiconductor technologies, accurate 3D-compositional and impurity analysis is an important must have since it plays a crucial role in the process development and fundamental understanding of novel devices and material properties. Atom probe tomography (APT) for that purpose is an important contender with its real 3D atomic-scale elemental analysis capability and excellent sensitivity $(<0.01$ at.\% [1]). However, the demanding metrology requirements of the semiconductor technology (spatial resolution, accuracy, sensitivity, reproducibility, heterogeneous systems, etc.) challenge the routine applicability of APT. Throughout an APT experiment numerous (atomistic) processes can be triggered before and during field evaporation (atom migration, retention, coevaporation), during the ions' flight sequence (dissociation, ion focusing) as well as during detection of the ions (multi-hits, mass overlap), which might result in compositional and spatial inaccuracies in the data. This paper provides a synopsis of our research projects that address these effects and artefacts.

For many semiconductor materials, accurate compositional analysis remains a challenge. A non-linear correlation between the quantification accuracy and the experimental condition, i.e. the electric field strength at the tip apex, is found in B-doped $\mathrm{Si}$ [2,3], $\mathrm{Si}_{0.75} \mathrm{Ge}_{0.25}$ [4], GaN [5-7], AlGaN [6,8], InGaAs [9]. Striving for an explanation, different root-causes should be considered involving element migration [10], DC evaporation, cluster dissociation with neutral formation [11,12], mass interference, multi-hit generation [2,13], etc. While they give a glimpse into biased loss (evaporation/detection) mechanisms, they fail to give a comprehensive picture or to predict the trends observed. In our studies $[3,4,7]$ the quantification accuracy improves when increasing the electric field strength, with the measured composition converging to the "real" value. This statement can yet not be generalized as in some reports [5,6] accurate quantification is confined to a narrow (medium) field range only. Moreover, high field strengths might reduce the effective detection efficiency due to increased multi-hit generation $[9,14]$ and/or DC field evaporation. The latter leads to a preferential loss of the elements with a lower evaporation field and/or deteriorates the signal-to-noise ratio. Hence the sensitivity will be degraded, which becomes a problem when quantifying dopant atoms $(<100)$ in next generation devices [1].

Akin to the quantification accuracy, the precision (reproducibility) is also of concern. A notorious example is the quantification of $\mathrm{B}$ (in $\mathrm{Si}$ ), for which a good accuracy was reported [15] whereas others observed an underestimate of $16 \%$ [3] up to $36 \%$ [2]. Similar discrepancies have been found on the apparent composition of $\mathrm{GaN}$ under identical field conditions [5-7]. Our recently proposed interlaboratory study (on B-doped SiGe, [16]) might help to better assess such discrepancies.

Although a correlation between the quantification accuracy and electric field strength has been shown, this becomes less trivial on the nm-scale. One cannot use the average field strength over the entire field of view but rather must consider the local electric field experienced by the local evaporation sites. In 
particular if one attempts to analyze spatially resolved compositional fluctuations, one of the strengths APT offers, local electric field variations in the vicinity of the poles [8] or at local protrusions on the tip will lead to inaccuracies in the apparent compositions. In addition, electric field disturbances around local protrusions might alter ion trajectories. If ion pathways cross each other, ions are falsely placed within the volume and the measured composition might be biased as well $[17,18]$. In these instances, neither quantification nor spatial accuracy is achieved.

Spatial inaccuracies are a notorious problem when analyzing heterogeneous systems. Root causes are the retention and migration of high field species on the tip surface (e.g. B in Si $[3,10])$ and the tendency of a heterogeneous tip to establish a non-hemispherical end shape during analysis [17]. This alters the local electric field distribution and hence ion trajectories, and results in local (de)magnification effects (unaccounted for in current data reconstruction algorithms). Knowledge on the (3D) tip shape is clearly a must, which led us to explore scanning probe microscopy [19] and photon-based imaging concepts [20]. Both might potentially be integrated into an atom probe microscope, thereby offering in theory insitu access to the tip shape. Obviously, these (real-time) tip shapes must be incorporated into a 3D data reconstruction algorithm, which is currently an important road blocker. Other challenges arise from the fact that non-detected atoms (limited field of view and detection efficiency) need to be placed back accurately in the reconstructed volume [21]. Yu-Ting [21] has recently pioneered an innovative approach that shall account for this (asymmetric tip shape, limited field of view and detection efficiency) though the computationally intense calculations limit its applicability to small systems $(<20 \mathrm{~nm})$.

In a nutshell, accurate and precise APT analysis in semiconductor applications can be hampered by various physical mechanisms. Understanding their impact can lead to an improved metrology generating relevant insight into material properties and fabrication processes.

References:

[1] A. J. Martin et al., Ultramicroscopy 186 (2018), p. 104.

[2] F. Meisenkothen et al., Ultramicroscopy 159 (2015), p. 101.

[3] D. Melkonyan et al., APT\&M 2018 (2018).

[4] M. Dialameh et al., APT\&M 2018 (2018).

[5] L. Mancini et al., The Journal of Physical Chemistry C 118 (2014), p. 24134.

[6] L. Rigutti et al., Scripta Materialia 148 (2018), p.81.

[7] R. J. H. Morris et al., Journal of Vacuum Science \& Technology B 36 (2018), p. 03 F130.

[8] R. J. H. Morris et al., APT\&M 2018 (2018).

[9] R. Cuduvally et al., APT\&M 2018 (2018).

[10] Y. Tu et al., Ultramicroscopy 173 (2017) p.58.

[11] B. Gault et al., New Journal of Physics 18 (2016), p. 033031.

[12] D. Zanuttini et al., Phys. Rev. A 95 (2017), p. 061401.

[13] Z. Peng et al., Ultramicroscopy 189 (2018) p. 54.

[14] M. Müller et al., Ultramicroscopy 111 (2011) p. 487.

[15] D. Kouzminov et al., APT\&M 2018 (2018).

[16] http://www.vamas.org/twa/projects.html (accessed February 22, 2019).

[17] D. Melkonyan et al., Ultramicroscopy 179 (2017), p. 100.

[18] C. Hatzoglou et al., Journal of Nuclear Materials 505 (2018), p. 240.

[19] C. Fleischmann et al., Ultramicroscopy 194 (2018), p. 221.

[20] P. van der Heide et al., M\&M 2019 (2019).

[21] Y.-T. Ling et al., APT\&M 2018 (2018). 